Short Note

\title{
Development and Characterization of New Single Nucleotide Polymorphism Markers from Expressed Sequence Tags in Common Carp (Cyprinus carpio)
}

Chuankun Zhu ${ }^{1,2}$, Lei Cheng ${ }^{1}$, Jingou Tong ${ }^{1, *}$ and Xiaomu Yu ${ }^{1}$

1 State Key Laboratory of Freshwater Ecology and Biotechnology, Institute of Hydrobiology, Chinese Academy of Sciences, Wuhan 430072, China; E-Mails: zhuchuankun@ihb.ac.cn (C.Z.); mailchenglei@gmail.com (L.C.); xmyu@ihb.ac.cn (X.Y.)

2 Graduate School of Chinese Academy of Sciences, Beijing 100039, China

* Author to whom correspondence should be addressed; E-Mail: jgtong@ihb.ac.cn; Tel.: +82-27-6878-0751; Fax: +82-27-6878-0123.

Received: 16 February 2012; in revised form: 17 May 2012 / Accepted: 22 May 2012 / Published: 14 June 2012

\begin{abstract}
The common carp (Cyprinus carpio) is an important aquaculture fish worldwide but only limited single nucleotide polymorphism (SNP) markers are characterized from expressed sequence tags (ESTs) in this species. In this study, 1487 putative SNPs were bioinformatically mined from 14,066 online ESTs mainly from the European common carp, with the occurrence rate of about one SNP every $173 \mathrm{bp}$. One hundred and twenty-one of these SNPs were selected for validation using PCR fragment sequencing, and 48 out of 81 primers could amplify the expected fragments in the Chinese common carp genome. Only 26 (21.5\%) putative SNPs were validated, however, 508 new SNPs and 68 indels were identified. The ratios of transitions to transversions were 1.77 for exon SNPs and 1.05 for intron SNPs. All the 23 SNPs selected for population tests were polymorphic, with the observed heterozygosity (Ho) ranging from 0.053 to 0.526 (mean 0.262 ), polymorphism information content (PIC) from 0.095 to 0.357 (mean 0.246), and 21 SNPs were in Hardy-Weinberg equilibrium. These results suggest that different common carp populations with geographic isolation have significant genetic variation at the SNP level, and these new EST-SNP markers are readily available for genetics and breeding studies in common carp.
\end{abstract}


Keywords: common carp (Cyprinus carpio); expressed sequence tag (EST); single nucleotide polymorphism (SNP); validation; genetic variations

\section{Introduction}

The common carp (Cyprinus carpio) is an important freshwater species for aquaculture and has been domesticated for $\mathrm{ca} .4000$ years [1]. Annual production of common carp has been increasing for 30 years, and recent production reached 3.3 million tons [2]. Numerous microsatellite markers [3-5] have been developed in common carp in response to the demands of genetic and breeding studies. Compared with microsatellites, single nucleotide polymorphisms (SNPs) are more promising molecular markers in genetics, genomics and aquaculture. Because of their high abundance in the genome, hereditable stability and allele portability, and the possibility for high-throughput analysis, they may replace traditional molecular markers in genetic studies [6,7]. EST-database mining is an efficient way to obtain SNP markers and was first applied for SNP discovery in human sequences [8], EST-SNPs were also obtained in many fish species [9-12], while to date only limited EST-SNPs have been characterized [13] in common carp. In this study, discovery of a set of EST-SNPs from online common carp EST databases and validation of selected EST-SNPs in the Chinese common carp were carried out, with the aims of (1) developing novel SNP resources for future studies of genetics and breeding in common carp; (2) validating and characterizing SNPs in common carp with different genetic backgrounds for the comparison of genetic variations.

\section{Results and Discussion}

The alignment of 14,066 online common carp ESTs resulted in the identification of 8862 unigenes (6727 singletons and 2135 contigs). The number of EST sequences per contig ranged from two to 60 (mean 3.4). Based on searches of the 2135 contigs, a total of 49,476 variations were detected, including 40,749 nucleotide substitutions and 8509 indels. After the additional filtering criterion was applied, a total of 1487 putative SNPs were obtained from 303 contigs, with an average frequency of one SNP per $173 \mathrm{bp}$ of contig sequence, which was greater than frequencies reported in Atlantic salmon (one SNP per 614 bp) [10], brown trout (one SNP per 463 bp) [14] and Pacific salmon (one SNP per 239 bp) [15], but close to the frequency reported in chum salmon (one SNP per $175 \mathrm{bp}$ ) [15]. The distribution of SNPs in these EST contigs ranged from one to 32 (Figure 1). Of the unigenes described in this study, 4127 (46.7\%) were annotated, and the annotations for the contigs containing valid SNPs in the Chinese common carp are listed in Table 1.

Of the 81 pairs of primers, 48 produced PCR products. Among these, 30 amplified products were larger than the expected size, indicating the existence of introns (Tables 1,S1). A total length of ca. 27,010 bp was amplified and sequenced, of which 13,213 bp were exons and 13,797 bp were introns. Finally, 121 SNPs were identified from 13,213 bp of exons, and 26 (21.5\%) of these putative SNPs were verified in a Chinese common carp population. On the other hand, a large number of new SNPs, which were not detected in the SNP discovery by data mining from the online EST sequences, were identified during the validation. These new SNPs included 202 SNPs and 10 indels in exons, and 
306 SNPs and 58 indels in introns (Table 1, Figure 2, Supplement Material 1). The distribution of SNPs in exon sequences ranged from one to 34 (Figure 1). In addition, the average rates of occurrence were about one SNP per 58 bp in the exon sequences, one SNP per 45 bp in the intron sequences, and one SNP per $53 \mathrm{bp}$ in the complete genomic sequences. These occurrence rate are much higher than the rates of putative SNPs discovered from the online EST databases in most fish species, but quite close to that of the hybridized catfish (1.32 SNP per $100 \mathrm{bp}$ ) [16]. In the present study, although the validation rate of predicted SNPs from online ESTs of European originated common carp was low, many new SNPs were found during the validation by sequencing the PCR products from the Chinese carp. These findings may suggest that different common carp breeds or geographic populations have different levels of genetic divergence and population structures, which are indicated by the gain or loss of SNP loci at a great level. However, these results should be interpreted with caution since the SNP frequency in discovery could be affected by many factors, such as sequencing depth, number of samples and population variation. Another factor that may be involved in this phenomenon is the higher rates of genome duplications in the common carp as indicated in other tetraploid fish such as some salmonids $[15,17,18]$.

Figure 1. Distribution of putative and actual single nucleotide polymorphisms (SNPs) per contig in common carp. (A) Distribution of putative SNPs discovered from online expressed sequence tags (ESTs); (B) Distribution of validated and new SNPs detected in the Chinese common carp ESTs.

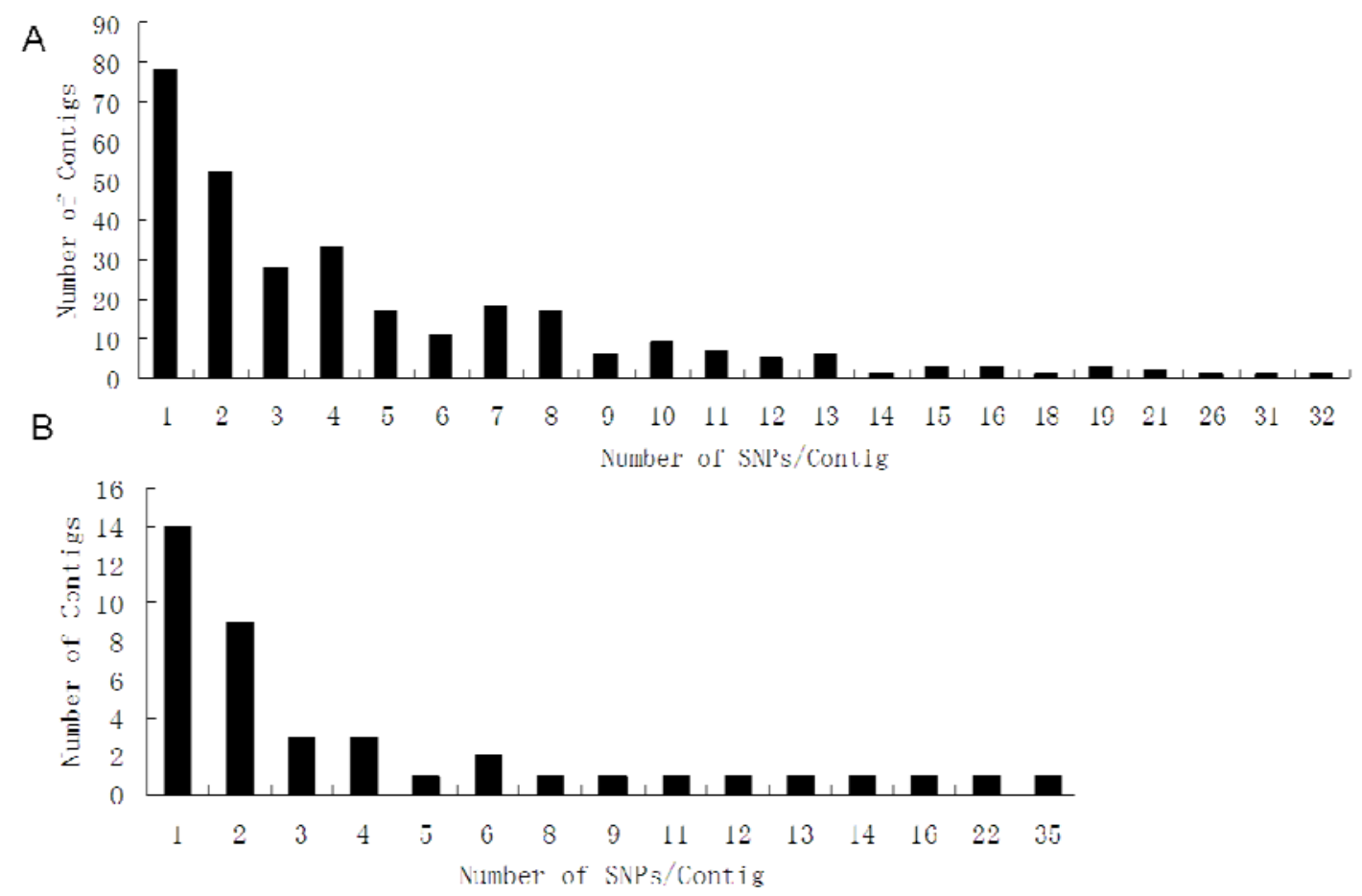

Among the 228 exon SNPs, 145 were transitions $(81 \mathrm{C} / \mathrm{T}, 64 \mathrm{~A} / \mathrm{G})$ and 82 were transversions $(27 \mathrm{~A} / \mathrm{C}, 19 \mathrm{C} / \mathrm{G}, 18 \mathrm{~A} / \mathrm{T}, 18 \mathrm{G} / \mathrm{T})$, while in intron SNPs there were 154 transitions and 149 transversions (Table 2). Two SNPs had both transition and transversion (C/G/T and A/G/T), this type of SNP has also been detected in Atlantic cod [11]. The ratio of transitions to transversions $\left(t_{\mathrm{s}} / \mathrm{t}_{\mathrm{v}}\right)$ was 1.77 for exon SNPs, 1.05 for intron SNPs and 1.31 for SNPs in complete genomic sequences. These 
ratios are similar to those reported in turbot (1.885) [12], chinook salmon (1.49) [15], gilthead seabream (1.375) [9] and zebrafish (1.20) [19]. However, in other fishes, such as chum salmon $\left(\mathrm{t}_{\mathrm{s}} / \mathrm{t}_{\mathrm{v}}=0.95\right)$ and sockeye salmon $\left(\mathrm{t}_{\mathrm{s}} / \mathrm{t}_{\mathrm{v}}=0.98\right)[15]$, the $\mathrm{t}_{\mathrm{s}} / \mathrm{t}_{\mathrm{v}}$ ratios were significantly lower, and only close to the ratio estimated from the intron regions of common carp ESTs (1.05) in this study. This discrepancy in $t_{s} / t_{v}$ ratios may suggest biased codon usage or substitution rate because fishes from different phylogenetic units may be subject to different selection pressures. Comparison of exon and intron SNPs revealed extreme differences in almost all aspects, including SNP frequency, $t_{\mathrm{s}} / \mathrm{t}_{\mathrm{v}}$ value, and commonest and rarest SNP types (Figure 2, Table 2). This may be explained by dissimilar natural selection pressures on exons and introns. In exons, most destructive mutations, which cause loss of protein function, vanish with less fit individuals, while beneficial mutations are retained and accumulate during evolution. Conversely, evolutionary constraints on introns are relatively weak, and more variations could be retained $[20,21]$.

Twenty-three SNPs were chosen to test the polymorphisms in 38 unrelated individuals from a Yangtze River common carp population. All these loci were found to be polymorphic, with frequencies of minor allele ranging from 0.053 to 0.368 (mean 0.19 ; Table 3 ), observed heterozygosity from 0.053 to 0.526 (mean 0.262; Table 3), and polymorphism information content (PIC) from 0.095 to 0.357 (mean 0.246; Table 3). In the exact test for HWE, 21 SNPs were in HWE $(p>0.05)$, and 2 SNPs deviated significantly from HWE after Bonferroni corrections $(p<0.00217)$. Linkage disequilibrium (LD) was detected between 19 pairs of the SNP loci after Bonferroni corrections $(p<0.00020)$.

Figure 2. Distribution of different kinds of nucleotide variances in exons (A), introns (B) and complete sequences (C) in successfully amplified sequences.

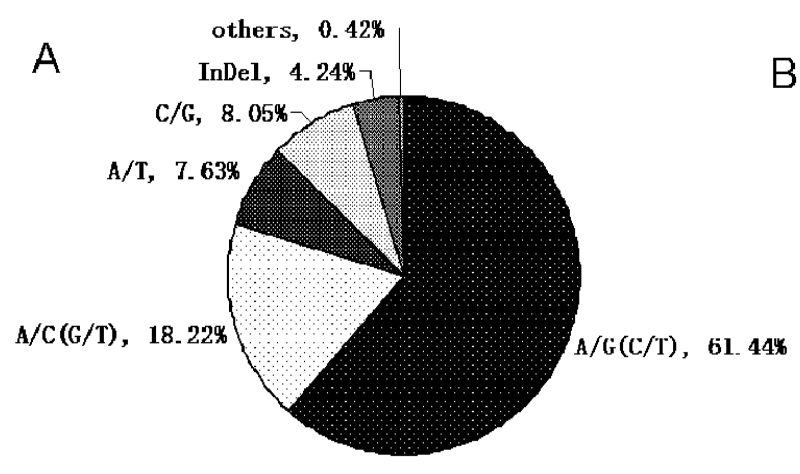

C

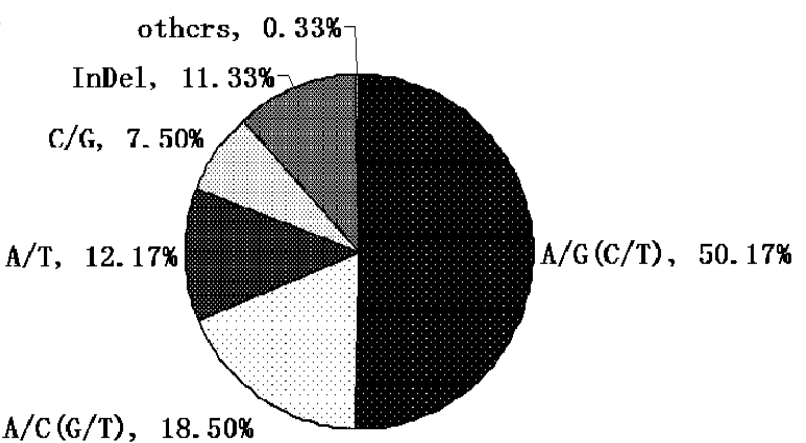

$\mathrm{A} / \mathrm{C}(\mathrm{G} / \mathrm{T}), 18.50 \%$

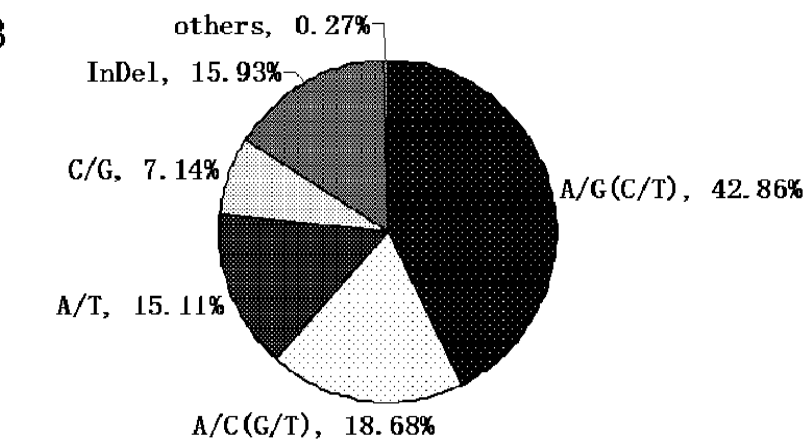

$\mathrm{A} / \mathrm{C}(\mathrm{G} / \mathrm{T}), \quad 18.68 \%$ 
Table 1. Validation of putative SNPs in the Chinese common carp and annotation for the SNP-containing EST sequences.

\begin{tabular}{|c|c|c|c|c|c|}
\hline Cluster ID & $\begin{array}{r}\text { Primers } \\
\text { designed }\end{array}$ & $\begin{array}{c}\text { Length (bp, } \\
\text { anticipative/actual) }\end{array}$ & $\begin{array}{c}\text { Number of SNPs } \\
\text { (putative/validated) }\end{array}$ & $\begin{array}{c}\text { New SNPs } \\
\text { in exon/intron }\end{array}$ & Annotation \\
\hline \multirow{2}{*}{ Cyprinus_Cluster7873.seq.Contig1 } & $7873-1$ & $180 / 388$ & $1 / 0$ & $14 / 32(5)^{\mathrm{a}}$ & \multirow{2}{*}{ extracellular space } \\
\hline & $7873-2$ & $196 / 313$ & $1 / 0$ & $4 / 0$ & \\
\hline Cyprinus_Cluster7877.seq.Contig1 & 7877 & $163 / 250$ & $6 / 1$ & $0 / 0$ & $\begin{array}{l}\text { malate dehydrogenase } \\
\text { (oxaloacetate-decarboxylating) (NADP+) activity }\end{array}$ \\
\hline Cyprinus_Cluster7885.seq.Contig1 & 7885 & $215 / 215$ & $1 / 0$ & $1(2) /-$ & actin binding \\
\hline Cyprinus_Cluster7889.seq.Contig1 & 7889 & $319 / 822$ & $1 / 0$ & $0 / 0$ & cornified envelope \\
\hline Cyprinus_Cluster7892.seq.Contig1 & 7892 & $253 / 377$ & $4 / 1$ & $0 / 0$ & structural constituent of ribosome \\
\hline Cyprinus_Cluster7895.seq.Contig1 & $7895-2$ & $157 / 954$ & $1 / 0$ & $2 / 11(5)$ & cerebroside-sulfatase activity \\
\hline Cyprinus_Cluster7896.seq.Contig1 & 7896 & $448 / 450$ & $1 / 0$ & $1 /-$ & nucleus \\
\hline Cyprinus_Cluster7898.seq.Contig1 & 7898 & $238 / 238$ & $0 / 0$ & $13 /-$ & creatine kinase activity \\
\hline Cyprinus_Cluster7909.seq.Contig1 & 7909 & $533 / 533$ & $2 / 0$ & $13 /-$ & N.A. ${ }^{b}$ \\
\hline Cyprinus_Cluster7918.seq.Contig1 & 7918 & $278 / 550$ & $1 / 1$ & $1 / 0$ & cathepsin $\mathrm{H}$ activity \\
\hline Cyprinus_Cluster7921.seq.Contig1 & 7921 & $164 / 319$ & $1 / 0$ & $1 / 2$ & N.A. \\
\hline Cyprinus_Cluster7927.seq.Contig1 & 7927 & $322 / 322$ & $1 / 1$ & $1 /-$ & N.A. \\
\hline Cyprinus_Cluster7929.seq.Contig1 & 7929 & $222 / 1433$ & $1 / 0$ & $1 / 13(3)$ & 3-hydroxyanthranilate 3,4-dioxygenase activity \\
\hline Cyprinus_Cluster7933.seq.Contig1 & 7933 & $429 / 1016$ & $1 / 1$ & $34(1) / 55(18)$ & glutathione transferase activity \\
\hline Cyprinus_Cluster7943.seq.Contig1 & 7943 & $402 / 405$ & $4 / 0$ & $1(2) /-$ & N.A. \\
\hline Cyprinus_Cluster7944.seq.Contig1 & 7944 & $298 / 298$ & $4 / 0$ & $5 /-$ & GTPase activity \\
\hline Cyprinus_Cluster7947.seq.Contig1 & 7947 & $236 / 419$ & $2 / 1$ & $2 / 3$ & lysozyme activity \\
\hline Cyprinus_Cluster7953.seq.Contig1 & 7953 & $347 / 347$ & $1 / 1$ & $7 /-$ & serine-type endopeptidase inhibitor activity \\
\hline Cyprinus_Cluster7955.seq.Contig1 & 7955 & $240 / 357$ & $3 / 0$ & $0 / 0$ & actin binding \\
\hline Cyprinus_Cluster7957.seq.Contig1 & 7957 & $354 / 1428$ & $5 / 0$ & $2 / 17(2)$ & fructose-bisphosphate ldolase activity \\
\hline Cyprinus_Cluster7961.seq.Contig1 & 7961 & $222 / 418$ & $2 / 1$ & $21 / 26(5)$ & external side of plasma membrane \\
\hline Cyprinus_Cluster7969.seq.Contig1 & 7969 & $217 / 216$ & $4 / 4$ & 7/- & antigen binding \\
\hline Cyprinus_Cluster7974.seq.Contig1 & 7974 & $215 / 443$ & $6 / 0$ & $1 / 5$ & N.A. \\
\hline
\end{tabular}


Table 1. Cont.

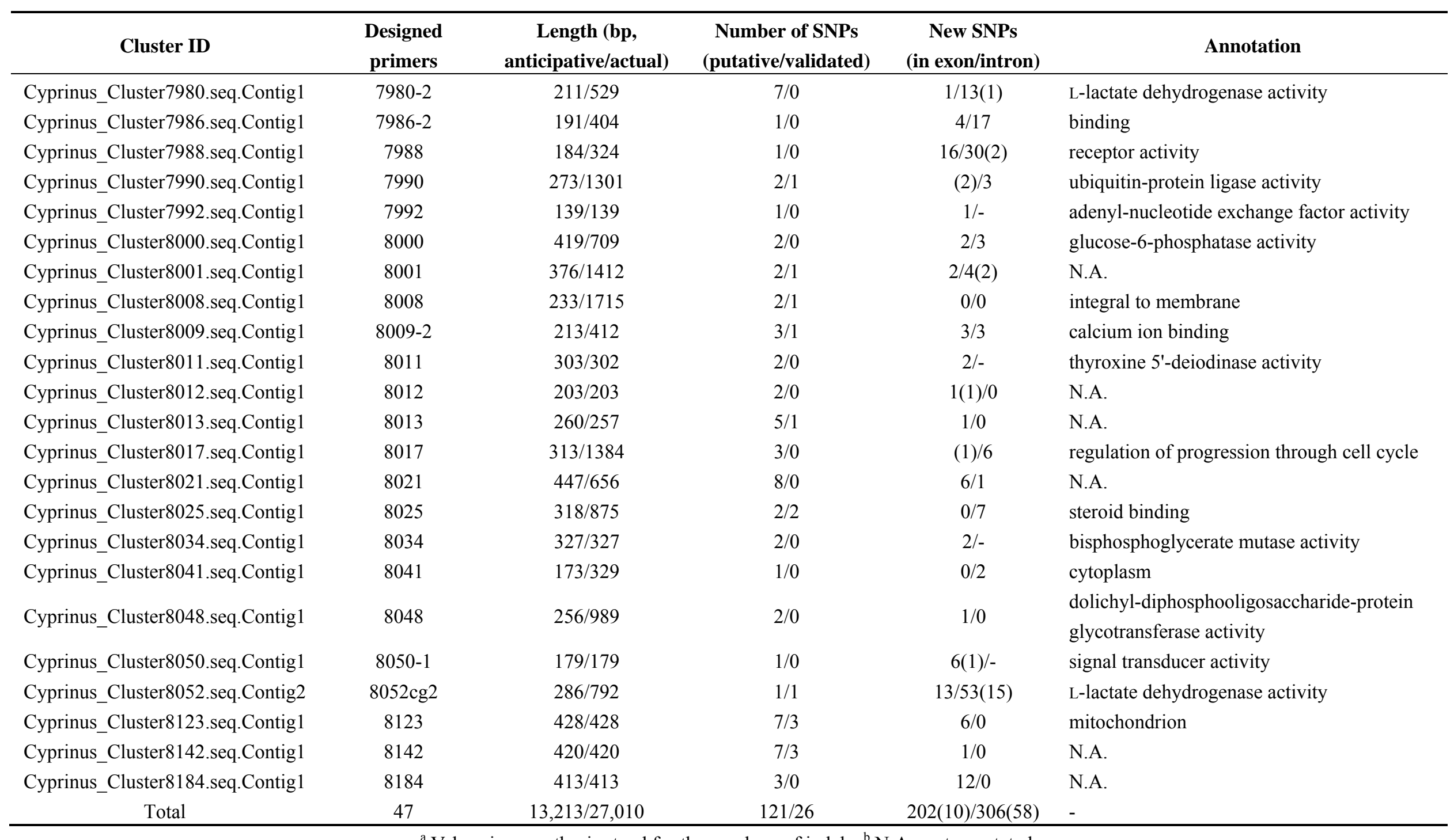

${ }^{\mathrm{a}}$ Values in parenthesis stand for the numbers of indels; ${ }^{\mathrm{b}}$ N.A.: not annotated. 
Table 2. Numbers of transitions and transversions in different genomic regions of common carp.

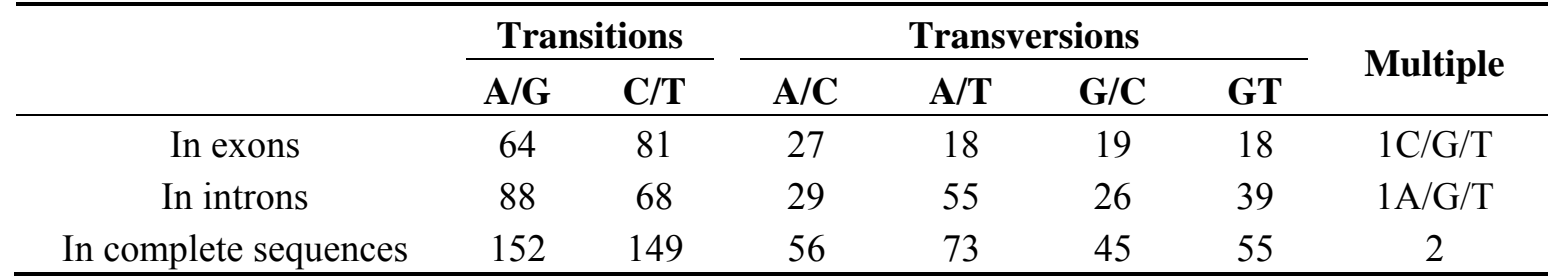

Table 3. Characterization of 23 polymorphic SNPs in a test population of the Chinese common carp.

\begin{tabular}{|c|c|c|c|c|c|}
\hline Loci & Allele Frequencies & $\boldsymbol{H}_{\mathrm{e}}$ & $\mathrm{H}_{\mathbf{0}}$ & PIC & $p$-value \\
\hline $\mathrm{CC}^{\mathrm{a}} 7892 \mathrm{G}>\mathrm{A}$ & $0.355(\mathrm{~A}) / 0.645(\mathrm{G})$ & 0.464 & 0.342 & 0.403 & 0.15446 \\
\hline $\mathrm{CC} 7953 \mathrm{C}>\mathrm{G}$ & $0.263(\mathrm{G}) / 0.737(\mathrm{C})$ & 0.393 & 0.526 & 0.313 & 0.04006 \\
\hline $\mathrm{CC} 7943 \mathrm{G}>\mathrm{A}$ & $0.316(\mathrm{~A}) / 0.684(\mathrm{G})$ & 0.438 & 0.474 & 0.339 & 0.71678 \\
\hline CC7909-1G $>A$ & $0.355(\mathrm{~A}) / 0.645(\mathrm{G})$ & 0.464 & 0.500 & 0.353 & 0.73065 \\
\hline $\mathrm{CC} 7909-2 \mathrm{~T}>\mathrm{C}$ & $0.145(\mathrm{C}) / 0.855(\mathrm{~T})$ & 0.251 & 0.237 & 0.217 & 0.57020 \\
\hline CC7909-3A > G & $0.25(\mathrm{G}) / 0.75(\mathrm{~A})$ & 0.380 & 0.395 & 0.305 & 1.00000 \\
\hline CC7909-4A > T & $0.145(\mathrm{~T}) / 0.855(\mathrm{~A})$ & 0.251 & 0.237 & 0.217 & 0.56984 \\
\hline $\mathrm{CC} 7909-5 \mathrm{~T}>\mathrm{C}$ & $0.145(\mathrm{C}) / 0.856(\mathrm{~T})$ & 0.251 & 0.237 & 0.217 & 0.56926 \\
\hline $\mathrm{CC} 7909-6 \mathrm{G}>\mathrm{C}$ & $0.145(\mathrm{C}) / 0.857(\mathrm{G})$ & 0.251 & 0.237 & 0.217 & 0.56908 \\
\hline CC7909-7T $>A$ & $0.092(\mathrm{~A}) / 0.908(\mathrm{~T})$ & 0.169 & 0.079 & 0.153 & 0.01852 \\
\hline CC7909-8A $>$ G & $0.079(\mathrm{G}) / 0.921(\mathrm{~A})$ & 0.147 & 0.105 & 0.135 & 0.19207 \\
\hline CC7909-9T $>$ A & $0.066(\mathrm{~A}) / 0.934(\mathrm{~T})$ & 0.125 & 0.079 & 0.115 & 0.13050 \\
\hline CC7909-10T $>C$ & $0.224(\mathrm{C}) / 0.776(\mathrm{~T})$ & 0.352 & 0.184 & 0.287 & 0.00768 \\
\hline CC7909-11T $>C$ & $0.092(\mathrm{C}) / 0.908(\mathrm{~T})$ & 0.169 & 0.079 & 0.153 & 0.01862 \\
\hline CC7909-12T $>$ A & $0.092(\mathrm{~A}) / 0.909(\mathrm{~T})$ & 0.169 & 0.079 & 0.153 & 0.01853 \\
\hline CC7909-13T $>C$ & $0.368(\mathrm{C}) / 0.632(\mathrm{~T})$ & 0.472 & 0.158 & 0.357 & 0.00003 \\
\hline CC7969-1G > T & $0.053(\mathrm{~T}) / 0.947(\mathrm{G})$ & 0.101 & 0.053 & 0.095 & 0.07912 \\
\hline CC7969-2C $>A$ & $0.290(\mathrm{~A}) / 0.710(\mathrm{C})$ & 0.417 & 0.158 & 0.327 & 0.00030 \\
\hline CC7969-3C $>$ G & $0.111(\mathrm{G}) / 0.889(\mathrm{C})$ & 0.200 & 0.111 & 0.178 & 0.04048 \\
\hline CC7969-4A $>$ G & $0.167(\mathrm{G}) / 0.833(\mathrm{~A})$ & 0.282 & 0.333 & 0.239 & 0.55952 \\
\hline CC7969-5A $>C$ & $0.236(\mathrm{C}) / 0.764(\mathrm{~A})$ & 0.366 & 0.472 & 0.296 & 0.15560 \\
\hline CC7969-6A $>$ G & $0.236(\mathrm{G}) / 0.764(\mathrm{~A})$ & 0.366 & 0.472 & 0.296 & 0.15479 \\
\hline CC7969-7T $>$ G & $0.236(\mathrm{G}) / 0.764(\mathrm{~T})$ & 0.366 & 0.472 & 0.296 & 0.15639 \\
\hline
\end{tabular}

${ }^{\mathrm{a}} \mathrm{CC}$ is the abbreviation of the cluster ID prefix "Cyprinus_Cluster".

\section{Experimental Section}

\subsection{Detection and Annotation of Putative SNPs}

A total of 14,192 EST sequences of common carp (mainly European breeds) were downloaded from GenBank, DNA Databank of Japan (DDBJ) and European Molecular Biology Laboratory (EMBL) databases. Sequences of length less than 100 bases were removed, and the remaining 14,066 EST sequences were used for further processing. Overlapping sequences were identified by cluster analysis using the UIcluster v. 2.02 software [22]. After this, the ESTs were divided into many clusters, and then alignment was performed using the Phrap program [23] for every cluster containing more than four sequences. SNPs were detected by the autoSNP program [24], with an additional criterion that the 
appearance of the minor allele of a given locus in the assembly of overlapping sequences must occur at least two times. Complete contigs containing SNPs were compared (BLASTX) to the UniProt_SWISSProt database and annotated with the top BLASTX hit if the database match had an $e$-value of $\leq 10^{-5}$. Matches to hypothetical gene and protein sequences were filtered out.

\subsection{Validation and Characterization of SNPs}

One hundred and ninety-seven SNPs (Supplement material 2) from 73 contigs were randomly selected for validation, and 81 pairs of primers were designed using the Primer 5 software to amplify the genomic fragments containing these SNPs via polymerase chain reaction (PCR). The validation panel contained eight individuals from three different common carp populations (breeds) (Yangtze River common carp $n=4$, Wuyuan (purse) red carp $n=2$, and Xingguo red carp $n=2$ ). A population of common carp with 38 unrelated individuals from the Zhangdu Lake of the Yangtze River, Wuhan, China, was used for polymorphism analysis of selected valid SNPs. Genome DNA was extracted from fin clips following the standard phenol-chloroform protocol [25].

PCR amplifications were carried out in a thermal cycler (MyCycler, BIO-RAD) in $60 \mu \mathrm{L}$ reaction volumes containing $6 \mu \mathrm{L}$ of $10 \times$ reaction buffer, $2 \mu \mathrm{L}$ of dNTP $(10 \mathrm{mmol} / \mathrm{l}), 2 \mathrm{U}$ of Taq polymerase (TIANGEN, China), $3.5 \mu \mathrm{L}$ of forward and reverse primer mixture $(2.5 \mathrm{mmol} / \mathrm{L}), 3.5 \mu \mathrm{L}$ of template DNA and $44 \mu \mathrm{L}$ of sterile water, using the following program: $94{ }^{\circ} \mathrm{C}$ for $5 \mathrm{~min}$, followed by 35 cycles of $94{ }^{\circ} \mathrm{C}$ for $40 \mathrm{~s}$, optimum annealing temperature for $40 \mathrm{~s}$, and $72{ }^{\circ} \mathrm{C}$ for $60 \mathrm{~s}$, and a final extension of $72{ }^{\circ} \mathrm{C}$ for $7 \mathrm{~min}$. PCR amplicons were purified using the Gel Extraction System B (BioDev-tech, China), according to the manufacturer's instructions. Purified PCR products were cloned into the PMD18-T vector and sequenced on an ABI 3730XL machine (Majorbio, China). For SNP genotyping, PCR-restricted fragment length polymorphism [26], direct sequencing and fragment length discrepant allele specific-PCR [27] were carried out for a given SNP.

\subsection{Data Analysis}

Alignment of the sequenced fragments was performed using ClustalX v. 1.81 [28], and putative SNP and small indels were detected using autoSNP program [24] and checked manually. Polymorphic indices were calculated using the Popgene v. 1.31 [29] and Excel Microsatellite Toolkit [30] software. The fitness to the Hardy-Weinberg equilibrium (HWE) at each locus, and pairwise linkage disequilibrium (LD) were tested for all validated SNPs using the Arlequin v. 3.1 software [31].

\section{Conclusions}

A total 1487 putative SNPs were identified by mining from online common carp EST sequences. Approximately 320 of the putative SNPs are expected to be true in the Chinese common carp as estimated based on the validation rate of $21.5 \%$ in this study. In spite of the low validation rate, large numbers of new SNPs were identified in the Chinese common carp. Of the 23 SNPs tested, all loci were polymorphic in a Yangtze River population with moderate diversity. These results indicate that the occurrence of SNPs varies significantly between European and Chinese populations, and these 
characterized SNPs are valuable resources for population genetics, high-resolution genetic maps, QTL (quantitative trait locus) identification, and maker assisted breeding in the common carp.

\section{Acknowledgements}

This work was partially supported by the MOST973 Program (2010CB126305), the MOA Special Programs (200903045 and 2011-G12) and the SKL-FEBL (2011FBZ20) of China. The authors thank L.S. Liu, W.J. Guo and F.Z. Zeng for sample preparation and laboratory technical assistance.

\section{References}

1. Wohlfarth, G.W. Heterosis for growth rate in common carp. Aquaculture 1993, 113, 31-46.

2. Food and Agriculture Organization of the United Nations Fisheries and Aquaculture Department. Fishery and Aquaculture Statistics, 2009; FAO: Rome, Italy, 2011. Available online: www.fao.org/fishery/statistics/global-aquaculture-production/en (accessed on 25 May 2012).

3. Crooijmans, R.; Bierbooms, V.; Komen, J.; Van, D.P.; Groenen, M. Microsatellite markers in common carp (Cyprinus carpio L.). Anim. Genet. 1997, 28, 129-134.

4. Wang, D.; Liao, X.; Cheng, L.; Yu, X.; Tong, J. Development of novel EST-SSR markers in common carp by data mining from public EST sequences. Aquaculture 2007, 271, 558-574.

5. Yue, G.H.; Ho, M.Y.; Orban, L.; Komen, J. Microsatellites within genes and ESTs of common carp and their applicability in silver crucian carp. Aquaculture 2004, 234, 85-98.

6. Liu, Z.J.; Cordes, J.F. DNA marker technologies and their applications in aquaculture genetics. Aquaculture 2004, 238, 1-37.

7. Garvin, M.R.; Saitoh, K.; Gharrett, A.J. Application of single nucleotide polymorphisms to non-model species: A technical review. Mol. Ecol. Resour. 2010, 10, 915-934.

8. Picoult-Newberg, L.; Ideker, T.E.; Pohl, M.G.; Taylor, S.L.; Donaldson, M.A.; Nickerson, D.A.; Boyce-Jacino1, M. Mining SNPs from EST databases. Genome Res. 1999, 9, 167-174.

9. Cenadelli, S.; Maran, V.; Bongioni, G.; Fusetti, L.; Parma, P.; Aleandri, R. Identification of nuclear SNPs in gilthead seabream. J. Fish Biol. 2007, 70, 399-405.

10. Hayes, B.; Laerdahl, J.K.; Lien, S.; Moen, T.; Berg, P.; Hindar, K.; Davidson, W.S.; Koop, B.F.; Adzhubei, A.; Hoyheim, B. An extensive resource of single nucleotide polymorphism markers associated with Atlantic salmon (Salmo salar) expressed sequences. Aquaculture 2007, 265, 82-90.

11. Hubert, S.; Bussey, J.T.; Higgins, B.; Curtis, B.A.; Bowman, S. Development of single nucleotide polymorphism markers for Atlantic cod (Gadus morhua) using expressed sequences. Aquaculture 2009, 296, 7-14.

12. Vera, M.; Álvarez-Dios, J.A.; Millán, A.; Pardo, B.G.; Bouza, C.; Hermida, M.; Fernández, C.; Herrán, R.; Molina-Luzón, M.J.; Martínez, P. Validation of single nucleotide polymorphism (SNP) markers from an immune expressed sequence tag (EST) turbot, Scophthalmus maximus, database. Aquaculture 2011, 313, 31-41.

13. Zheng, X.; Kuang, Y.; Zhang, X.; Lu, C.; Cao, D.; Li, C.; Sun, X. A genetic linkage map and comparative genome analysis of common carp (Cyprinus carpio L.) using microsatellites and SNPs. Mol. Genet. Genomics 2011, doi:10.1007/s00438-011-0644-x. 
14. Nicod, J.C.; Largiadèr, C.R. SNPs by AFLP (SBA): A rapid SNP isolation strategy for non-model organisms. Nucleic Acids Res. 2003, 31, doi:10.1093/nar/gng019.

15. Smith, C.T.; Elfstrom, C.M.; Seeb, L.W.; Seeb, J.E. Use of sequence data from rainbow trout and Atlantic salmon for SNP detection in Pacific salmon. Mol. Ecol. 2005, 14, 4193-4203.

16. He, C.; Chen, L.; Simmons, M.; Li, P.; Kim, S.; Liu, Z.J. Putative SNP discovery in interspecific hybrids of catfish by comparative EST analysis. Anim. Genet. 2003, 34, 445-448.

17. Larhammar, D.; Risinger, C. Molecular genetic aspects of tetraploidy in the common carp Cyprinus carpio. Mol. Phylogenet. Evol. 1994, 3, 59-68.

18. David, L.; Blum, S.; Feldman, M.W.; Lavi, U.; Hillel, J. Recent duplication of the common carp (Cyprinus carpio L.) genome as revealed by analyses of microsatellite loci. Mol. Biolo. Evol. 2003, 20, 1425-1434.

19. Stickney, H.L.; Schmutz, J.; Woods, I.G.; Holtzer, C.C.; Dickson, M.C.; Kelly, P.D.; Myers, R.M.; Talbot, W.S. Rapid mapping of zebrafish mutations with SNPs and oligonucleotide microarrays. Genome Res. 2002, 12, 1929-1934.

20. Andolfatto, P. Adaptive evolution of non-coding DNA in Drosophila. Nature 2005, 437, 1149-1152.

21. Lynch, M.; Koskella, B.; Schaack, S. Mutation pressure and the evolution of organelle genomic architecture. Science 2006, 311, 1727-1730.

22. Pedretti, K.; Scheetz, T.; Braun, T.; Roberts, C.; Robinson, N.; Casavant, T. A Parallel Expressed Sequence Tag (EST) Clustering Program. In Proceedings of the 6th International Conference on Parallel Computing Technologies; Malyshkin, V., Ed.; Springer-Verlag Berlin: Novosibirsk, Russia, 2001; pp. 490-497.

23. Gordon, D.; Abajian, C.; Green, P. Consed: A graphical tool for sequence finishing. Genome Res. 1998, 8, 195-202.

24. Barker, G.; Batley, J.; O’Sullivan, H.; Edwards, K.J.; Edwards, D. Redundancy based detection of sequence polymorphisms in expressed sequence tag data using autoSNP. Bioinformatics 2003, 19, $421-422$.

25. Sambrook, J.; Russell, D.W. Molecular Cloning: A Laboratory Manual; Cold Spring Harbor Laboratory: Cold Spring Harbor, NY, USA, 2001; pp. 463-465.

26. Gallagher, P.M.; Lowe, G.; Fitzgerald, T.; Bella, A.; Greene, C.M.; McElvaney, N.G.; O'Neill, S.J. Association of IL-10 polymorphism with severity of illness in community acquired pneumonia. Thorax 2003, 58, 154-156.

27. Gaudet, M.; Fara, A.-G.; Sabatti, M.; Kuzminsky, E.; Mugnozza, G.S. Single-reaction for SNP genotyping on agarose gel by allele-specific PCR in black poplar (Populus nigra L.). Plant Mol. Biol. Rep. 2007, 25, 1-9.

28. Thompson, J.; Gibson, T.; Plewniak, F.; Jeanmougin, F.; Higgins, D. The CLUSTAL_X windows interface: Flexible strategies for multiple sequence alignment aided by quality analysis tools. Nucleic Acids Res. 1997, 25, 4876-4882.

29. Yeh, F.C.; Boyle, T. POPGENE: Microsoft Window-based Freeware for Population Genetic Analysis, version 1.31; University of Alberta: Edmonton, Canada, 1999.

30. Park, S.D.E. Trypanotolerance in West African Cattle and the Population Genetic Effects of Selection. Ph.D. Dissertation, University of Dublin: Dublin, Ireland, 2001. 
31. Excoffier, L.; Laval, G.; Schneider, S. ARLEQUIN: An integrated software package for population genetics data analysis. Version 3. Evol. Bioinform. Online 2005, 1, 47-50.

(C) 2012 by the authors; licensee MDPI, Basel, Switzerland. This article is an open access article distributed under the terms and conditions of the Creative Commons Attribution license (http://creativecommons.org/licenses/by/3.0/). 